\title{
Default Mode of Brain Function in Monkeys
}

\author{
Dante Mantini, ${ }^{1 \star}$ Annelis Gerits, ${ }^{1,2,3 *}$ Koen Nelissen, ${ }^{1}$ Jean-Baptiste Durand, ${ }^{1}$ Olivier Joly, ${ }^{1}$ Luciano Simone, ${ }^{1}$ \\ Hiromasa Sawamura, ${ }^{1}$ Claire Wardak, ${ }^{1}$ Guy A. Orban, ${ }^{1}$ Randy L. Buckner, ${ }^{2,3,4,5}$ and Wim Vanduffel ${ }^{1,2,3}$ \\ ${ }^{1}$ Laboratory for Neuro-psychophysiology, K.U. Leuven Medical School, Leuven 3000, Belgium, ${ }^{2}$ Athinoula A. Martinos Center for Biomedical Imaging, \\ Charlestown, Massachusetts 02129, Departments of ${ }^{3}$ Radiology and ${ }^{4}$ Psychiatry, Harvard Medical School, Boston, Massachusetts 02115, and ${ }^{5}$ Department \\ of Psychology, Center for Brain Science, Harvard University, Cambridge, Massachusetts 02138
}

Human neuroimaging has revealed a specific network of brain regions — the default-mode network (DMN) — that reduces its activity during goal-directed behavior. So far, evidence for a similar network in monkeys is mainly indirect, since, except for one positron emission tomography study, it is all based on functional connectivity analysis rather than activity increases during passive task states. Here, we tested whether a consistent DMN exists in monkeys using its defining property. We performed a meta-analysis of functional magnetic resonance imaging data collected in 10 awake monkeys to reveal areas in which activity consistently decreases when task demands shift from passive tasks to externally oriented processing. We observed task-related spatially specific deactivations across 15 experiments, implying in the monkey a functional equivalent of the human DMN. We revealed by resting-state connectivity that prefrontal and medial parietal regions, including areas 9/46d and 31, respectively, constitute the DMN core, being functionally connected to all other DMN areas. We also detected two distinct subsystems composed of DMN areas with stronger functional connections between each other. These clusters included areas 24/32, 8b, and TPOC and areas 23, v23, and PGm, respectively. Such a pattern of functional connectivity largely fits, but is not completely consistent with anatomical tract tracing data in monkeys. Also, analysis of afferent and efferent connections between DMN areas suggests a multisynaptic network structure. Like humans, monkeys increase activity during passive epochs in heteromodal and limbic association regions, suggesting that they also default to internal modes of processing when not actively interacting with the environment.

\section{Introduction}

Human neuroimaging studies revealed robust increases in cortical activity during passive tasks in contrast to epochs when individuals are engaged in externally oriented decisions (Shulman et al., 1997; Mazoyer et al., 2001; Laird et al., 2009). The ensemble of brain regions active during passive tasks, called the default-mode network (DMN), consistently includes the anterior and posterior cingulate cortex, medial and lateral parietal cortex, and medial prefrontal cortex (Raichle et al., 2001; Buckner et al., 2008). These regions form nexuses of distributed connectivity throughout heteromodal cortex (Greicius et al., 2003; Buckner et al., 2009) and possess metabolic properties that set them apart from other regions (Raichle et al., 2001; Vaishnavi et al., 2010). In recent years,

\footnotetext{
Received May 10, 2011; revised July 1, 2011; accepted July 20, 2011.

Author contributions: G.A.O., R.L.B., and W.V. designed research; D.M. and A.G. performed research; D.M. contributed unpublished reagents/analytic tools; D.M., A.G., K.N., J.-B.D., O.J., L.S., H.S., and C.W. analyzed data; D.M., R.L.B., and W.V. wrote the paper.

D.M. and K.N. are postdoctoral fellows of the Fonds Wetenschappelijk Onderzoek-Vlaanderen (F.W.O.). This work received support from European Union Grant Brainsync FP7 FWP-200728, Inter University Attraction Pole 6/29, Programme Financing PFV/10/008, Geconcerteerde Onderzoeks Actie 10/19, Hercules II funds, Fonds Wetenschappelijk Onderzoek-Vlaanderen Grants G062208N10 and G083111N10, and National Science Foundation Grant BCS0745436. The Martinos Center is supported by National Center for Research Resources Grant P41RR14075. We thank A. Coeman, C. Fransen, M. Depaep, W. Depuydt, P. Kayenbergh, G. Meulemans, and S. Verstraeten for technical assistance, Prof. A. Laird for the human data, and Prof. R. Tootell for his comments on the manuscript.

*D.M. and A.G. contributed equally to this work.

Correspondence should be addressed to Dr. Wim Vanduffel, Athinoula A. Martinos Center for Biomedical Imaging, 13th Street, Building 149, Charlestown, MA 02129. E-mail: wim@nmr.mgh.harvard.edu.

DOI:10.1523/JNEUROSCI.2318-11.2011

Copyright $\odot 2011$ the authors $\quad 0270-6474 / 11 / 3112954-09 \$ 15.00 / 0$
}

the DMN has attracted considerable interest, as well as controversy, mainly in relation to its functions.

To test specific hypotheses about the functional role of the DMN, it is important to clarify whether default mode activity is present in other primate species. Also, the definition of the DMN in monkeys would permit the investigation of its single-cell properties and the use of invasive interventional protocols to probe its functional role-which is less feasible in humans (Tsao et al., 2006; Ekstrom et al., 2008; Moeller et al., 2008; Caggiano et al., 2009). Despite the absence of linguistic abilities and presumably certain forms of self-referential processing (Passingham, 2009), many protoforms of human skills can be found in monkeys, thus allowing for comparative monkey-human studies for a wide range of tasks (Nakahara et al., 2002; Vanduffel et al., 2002; Orban et al., 2004; Tsao et al., 2008). Cytoarchitectonic maps in the vicinity of the human DMN have revealed anatomical similarities between humans and monkeys (Parvizi et al., 2006; Vogt et al., 2006; Buckner et al., 2008; Binder et al., 2009), supporting the notion that elements of the DMN may be conserved across primate species, although analogies for regions including the inferior parietal lobule are less certain (Caminiti et al., 2010). Notably, activity reductions under specific tasks were recently documented in monkeys by single-cell recordings in the posterior cingulate (Hayden et al., 2009) and by positron emission tomography (PET) in medial prefrontal and parietal areas (Kojima et al., 2009). Furthermore, functional connectivity investigations in anesthetized monkeys provide evidence for a network of regions in the monkey that may be a functional equivalent of 
Table 1. Details of the monkey fMRI datasets used for the study

\begin{tabular}{|c|c|c|c|}
\hline Experiment & Reference study & Key scanning parameters & Monkey (runs) \\
\hline \multicolumn{4}{|l|}{ Visual experiments } \\
\hline Adaptation & Sawamura et al., 2005 & 1.5 T scanner, 32 slices, voxel size $2 \times 2 \times 2 \mathrm{~mm}$, TE $27 \mathrm{~ms}$, TR $2.4 \mathrm{~s}$ (no gap), task/rest blocks $120 / 72 \mathrm{~s}$, run duration $384 \mathrm{~s}$ & $\begin{array}{l}\text { M5 (25) } \\
\text { M6 (25) }\end{array}$ \\
\hline Size invariance & Sawamura et al., 2005 & 1.5 T scanner, 32 slices, voxel size $2 \times 2 \times 2 \mathrm{~mm}$, TE $27 \mathrm{~ms}$, TR $2.4 \mathrm{~s}$ (no gap), task/rest blocks $180 / 36 \mathrm{~s}$, run duration $432 \mathrm{~s}$ & $\begin{array}{l}\text { M1 (25) } \\
\text { M5 (25) }\end{array}$ \\
\hline Eccentricity & Nelissen et al., 2006 & 1.5 T scanner, 32 slices, voxel size $2 \times 2 \times 2 \mathrm{~mm}$, TE $27 \mathrm{~ms}$, TR $2.4 \mathrm{~s}$ (no gap), task/rest blocks $96 / 24 \mathrm{~s}$, run duration $360 \mathrm{~s}$ & $\begin{array}{l}\text { M1 (20) } \\
\text { M5 (20) }\end{array}$ \\
\hline Opponent motion & Nelissen et al., 2006 & 1.5 T scanner, 32 slices, voxel size $2 \times 2 \times 2 \mathrm{~mm}$, TE $27 \mathrm{~ms}$, TR $2.4 \mathrm{~s}$ (no gap), task/rest blocks $96 / 24 \mathrm{~s}$, run duration $480 \mathrm{~s}$ & $\begin{array}{l}\text { M1 (15) } \\
\text { M3 (12) }\end{array}$ \\
\hline Optic flow & Nelissen et al., 2006 & 1.5 T scanner, 32 slices, voxel size $2 \times 2 \times 2 \mathrm{~mm}$, TE $27 \mathrm{~ms}$, TR $2.4 \mathrm{~s}$ (no gap), task/rest blocks $144 / 36$ s run duration $540 \mathrm{~s}$, & $\begin{array}{l}\text { M1 (10) } \\
\text { M4 (8) } \\
\text { M5 (10) }\end{array}$ \\
\hline Structure from motion & Durand et al., 2007 & 1.5 T scanner, 32 slices, voxel size $2 \times 2 \times 2 \mathrm{~mm}$, TE $27 \mathrm{~ms}$, TR $2.4 \mathrm{~s}$ (no gap), task/rest blocks $180 / 36 \mathrm{~s}$, run duration $432 \mathrm{~s}$ & $\begin{array}{l}\text { M1 (16) } \\
\text { M3 (16) } \\
\text { M5 (16) }\end{array}$ \\
\hline Stereo vision & Durand et al., 2007 & 1.5 T scanner, 32 slices, voxel size $2 \times 2 \times 2 \mathrm{~mm}$, TE $27 \mathrm{~ms}$, TR $2.4 \mathrm{~s}$ (no gap), task/rest blocks $180 / 36 \mathrm{~s}$, run duration $432 \mathrm{~s}$ & $\begin{array}{l}\text { M1 (16) } \\
\text { M3 (16) } \\
\text { M5 (16) }\end{array}$ \\
\hline Shading & Nelissen et al., 2009 & 1.5 T scanner, 32 slices, voxel size $2 \times 2 \times 2 \mathrm{~mm}$, TE $27 \mathrm{~ms}$, TR $2.4 \mathrm{~s}$ (no gap), task/rest blocks $180 / 36 \mathrm{~s}$, run duration $432 \mathrm{~s}$ & $\begin{array}{l}\text { M1 (16) } \\
\text { M3 (16) } \\
\text { M5 (16) }\end{array}$ \\
\hline \multicolumn{4}{|l|}{ Attention experiments } \\
\hline Visual search & Wardak et al., 2010 & 1.5 T scanner, 32 slices, voxel size $2 \times 2 \times 2 \mathrm{~mm}$, TE $27 \mathrm{~ms}$, TR $2.4 \mathrm{~s}$ (no gap), task/rest blocks $72 / 24 \mathrm{~s}$, run duration $384 \mathrm{~s}$ & $\begin{array}{l}\text { M9 (30) } \\
\text { M10 (30) }\end{array}$ \\
\hline Pop-out & Wardak et al., 2010 & 1.5 T scanner, 32 slices, voxel size $2 \times 2 \times 2 \mathrm{~mm}$, TE $27 \mathrm{~ms}$, TR $2.4 \mathrm{~s}$ (no gap), task/rest blocks $72 / 24 \mathrm{~s}$, run duration $384 \mathrm{~s}$ & $\begin{array}{l}\text { M9 (30) } \\
\text { M10 (30) }\end{array}$ \\
\hline \multicolumn{4}{|c|}{ Action-observation experiments } \\
\hline Human action & Nelissen et al., 2005 & 1.5 T scanner, 32 slices, voxel size $2 \times 2 \times 2 \mathrm{~mm}$, TE $27 \mathrm{~ms}$, TR $2.4 \mathrm{~s}$ (no gap), task/rest blocks $108 / 36 \mathrm{~s}$, run duration $432 \mathrm{~s}$ & $\begin{array}{l}\text { M1 (27) } \\
\text { M3 (18) } \\
\text { M5 (9) }\end{array}$ \\
\hline Action-moving object & Nelissen et al., 2005 & 1.5 T scanner, 32 slices, voxel size $2 \times 2 \times 2 \mathrm{~mm}$, TE $27 \mathrm{~ms}$, TR $2.4 \mathrm{~s}$ (no gap), task/rest blocks $108 / 36 \mathrm{~s}$, run duration $432 \mathrm{~s}$ & $\begin{array}{l}\text { M3 (20) } \\
\text { M5 (20) }\end{array}$ \\
\hline Tool-use observation & Peeters et al., 2009 & 1.5 T scanner, 32 slices, voxel size $2 \times 2 \times 2 \mathrm{~mm}$, TE $27 \mathrm{~ms}$, TR $2.4 \mathrm{~s}$ (no gap), task/rest blocks $76.8 / 19.2 \mathrm{~s}$, run duration $384 \mathrm{~s}$ & $\begin{array}{l}\text { M6 (48) } \\
\text { M13 (48) } \\
\text { M14 (48) }\end{array}$ \\
\hline \multicolumn{4}{|l|}{ Auditory experiments } \\
\hline $\begin{array}{l}\text { Monkey-human } \\
\text { sounds }\end{array}$ & Joly et al., 2011 & 1.5 T scanner, 32 slices, voxel size $2 \times 2 \times 2 \mathrm{~mm}$, TE $27 \mathrm{~ms}$, TR 5.0 s ( $56 \%$ gap), task/rest blocks $240 / 40$ s, run duration $560 \mathrm{~s}$ & $\begin{array}{l}\text { M13 (28) } \\
\text { M14 (28) } \\
\text { M18 (28) }\end{array}$ \\
\hline Scrambled sounds & Joly et al., 2011 & 1.5 T scanner, 32 slices, voxel size $2 \times 2 \times 2 \mathrm{~mm}$, TE $27 \mathrm{~ms}$, TR 5.0 s ( $56 \%$ gap), task/rest blocks $240 / 40$ s, run duration $560 \mathrm{~s}$ & $\begin{array}{l}\text { M13 (28) } \\
\text { M14 (28) } \\
\text { M18 (28) }\end{array}$ \\
\hline $\begin{array}{l}\text { Resting-state experiment } \\
\text { Continuous fixation }\end{array}$ & - & 3.0 T scanner, 40 slices, voxel size $1.25 \times 1.25 \times 1.25 \mathrm{~mm}$, TE $19 \mathrm{~ms}$, TR 2.0 s (no gap), steady state, run duration $600 \mathrm{~s}$ & $\begin{array}{l}\text { M13 (20) } \\
\text { M14 (20) } \\
\text { M18 (20) } \\
\text { M19 (20) }\end{array}$ \\
\hline
\end{tabular}

For each monkey fMRI dataset, the experimental task, the reference study, the main acquisition parameters, and the number of monkeys and acquisition runs are provided.

the human DMN (Vincent et al., 2007, 2010; Margulies et al., 2009).

However, the defining functional property of the DMN - that it increases activity in passive states relative to active periods of engagement with the environment- has not been explored in a manner parallel to the work in humans. Here we conducted an extensive meta-analysis of monkey fMRI data collected under many experimental conditions to investigate whether a consistent network of regions-a default mode network-is present in macaques. Following identification of a DMN in the monkey, we analyzed its network structure in a task-independent manner using resting-state connectivity. Furthermore, we compared the resting-state connectivity results with published anatomical connectivity data from tracing studies in the monkey.

\section{Materials and Methods}

Task experiments and data collection. Animal care met the Belgian and European guidelines and was approved by the K.U. Leuven Medical School. Experimental procedures were approved by the local Ethics Committees of the K.U. Leuven. Task-related fMRI data were acquired between 2003 and 2007 during 15 experiments conducted in our laboratory (Nelissen et al., 2005, 2006, 2009; Sawamura et al., 2005; Durand et al., 2007; Peeters et al., 2009; Wardak et al., 2010; Joly et al., 2011) (Table 1). They were collected in one female and nine male monkeys with a 1.5 T MR scanner (Siemens Sonata). All experiments followed a block design with fixation as control condition. The active task varied across experiments and belonged to one of the following groups of experiments: visual, attention, action-observation, and auditory. The resting-state fMRI data were collected in four fixating monkeys (three male and one female) during the years 2008-2009 with a 3.0 T MR scanner (Siemens Trio). 
Before scanning, a contrast agent (MION or Sinerem) was injected into the animal's femoral or saphenous vein (Vanduffel et al., 2001). Eye position was monitored using a pupil-corneal reflection tracking system (RK-726PCI, Iscan) at $60 \mathrm{~Hz}$. Monkeys were rewarded for fixation on a small red target $(0.30 \times 0.35$ visual degrees) in the middle of the screen (Vanduffel et al., 2001, 2002). The dimension of the fixation window was $2 \times 3$ visual degrees. The functional volumes were gradient-echo-planar images (GE-EPI) covering the whole brain.

Data preprocessing. fMRI time series were preprocessed and analyzed using the SPM5.0 toolbox (Wellcome Trust Centre for Neuroimaging). Spatial preprocessing consisted of realignment and rigid coregistration with a template anatomy [monkey M12 = MM1 from Ekstrom et al. (2008), $0.35 \mathrm{~mm}$ isotropic voxels]. To compensate for EPI distortions and interindividual anatomical differences, functional images were warped by a nonrigid matching technique (Nelissen et al., 2005, 2006). The monkey F99 image was used to define the target stereotaxic space. The images were resliced to $1 \mathrm{~mm}$ isotropic voxels and smoothed with an isotropic Gaussian kernel at $2 \mathrm{~mm}$ FWHM. The resting-state fMRI data were additionally preprocessed in preparation for functional connectivity analysis, according to the procedure described by Vincent et al. (2007). The preprocessing steps included the following: (1) bandpass filtering between 0.0025 and $0.05 \mathrm{~Hz}$; (2) regression of white matter and ventricle signals, and their first derivatives; and (3) regression of three-dimensional motion parameters, and their first derivatives.

Meta-analysis of task fMRI data. A mask image capturing the full extent of the template brain was used for General Linear Model (GLM) estimation. Head-realignment and eyemovement parameters were included in the model as covariates of no interest. Experiment-specific deactivation maps were delineated by contrasting the $\beta$-value map in the fixation condition with the $\beta$-value map in all the active-task conditions. Statistical $t$-score maps were obtained from the $\beta$-value maps by fixed-effect analysis. A consensus map for deactivations across experiments was created by binarizing the deactivation maps from single experiments $(p<$ 0.01 , uncorrected), summing these binarized masks, and thresholding by number of subjects $(N \geq 8)$.

Definition of DMN areas. Since the monkey DMN was defined though a meta-analysis across multiple subjects and experiments, uncertainty in the exact definition of included areas is possible, due to the alignment of multiple datasets to a template space and because fMRI responses often include regions that span multiple areas. The monkey DMN regions were determined by selecting local maxima (identified by their stereotaxic coordinates) in the consensus map. Each peak was projected on the anatomical image of the template (monkey F99) using the MRIcron software, and classified by comparing its position with respect to the monkey atlas of Paxinos et al. (2008). We opted to use this atlas because it already has an extended group of users (Frey et al., 2011). Subsequently, the peak and the monkey DMN map were projected together on the anatomical template image to evaluate the contribution of regions adjacent to the DMN peak. In cases where the extended region of deactivation clearly invaded neighboring monkey areas in the atlas, the classification was adapted accordingly and uncertainty acknowledged.

Functional connectivity analysis of resting-state fMRI data. Regions of interest (ROIs) in the monkey DMN were generated using the classified monkey DMN

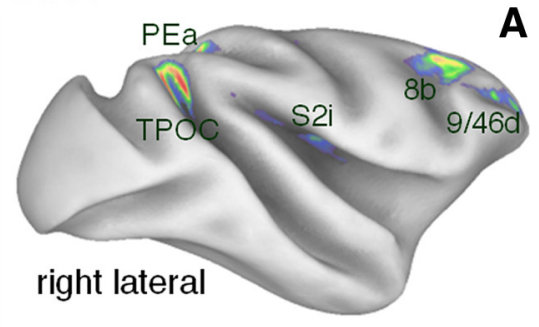

left medial

right medial

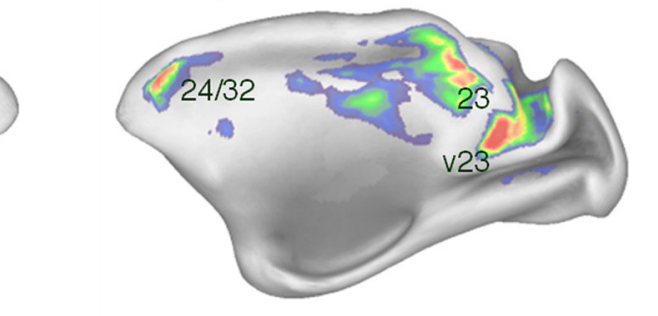

A

\section{ron}

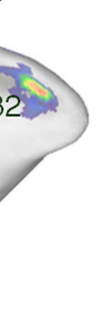

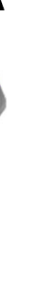
.

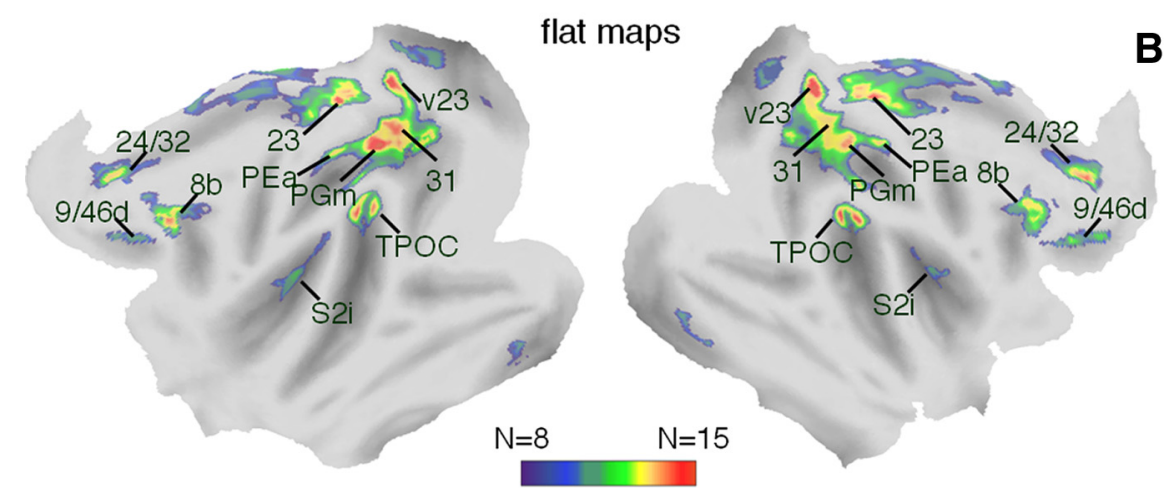

B

Figure 1. Network of consistent task-related deactivations in the monkey brain. $\boldsymbol{A}, \boldsymbol{B}$, Brain areas deactivated $(p<0.01)$ in at least 8 of the 15 monkey task-related experiments are illustrated in inflated cortical representations (lateral and medial views) $(\boldsymbol{A})$ and on flat maps ( $\boldsymbol{B})$. The areas are labeled on the flat maps on the basis of the monkey brain atlas by Paxinos et al. (2008).

DMN peaks. They were defined as spheres of $2 \mathrm{~mm}$ radius, centered on the respective stereotaxic coordinates. For each ROI, a representative time course was obtained by averaging the signals across voxels. Wholebrain connectivity maps were created and converted to $Z$-scores by the Fisher's $r$-to- $z$ transformation (Zar, 1998), correcting the degrees of freedom for the autocorrelation in the time-series (Shumway and Stoffer, 2006). A fixed-effect analysis was used to create group-level correlation maps (Genest, 1992). Finally, the group $Z$-score maps were backconverted to correlation maps by the Fisher's $z$-to- $r$ transformation (Zar, 1998).

Following the same statistical approach, a correlation matrix between the ROI time courses was calculated. ROIs without any significant correlation to other ROIs $(p<0.001)$ were excluded from subsequent analyses. A residual (unthresholded) correlation matrix between correlated ROIs was used for a network analysis across DMN nodes (AndrewsHanna et al., 2010). The goal of this analysis was to determine from the pairwise regional correlations whether the DMN areas clustered into coherent subsystems (Buckner et al., 2008). Graph analysis of the correlation matrix was implemented using the Kamada-Kawai algorithm, a spring-embedded algorithm that pulls connected nodes together and pushes disconnected nodes apart in a manner such that the total energy of the system is minimized (Kamada and Kawai, 1989). Network hubs, defined as nodes connected to most of the other nodes, were identified by means of the betweenness-centrality. This is a quantitative measure of how connected a particular node is to other nodes (Freeman, 1977). The nonhub nodes, connected by definition to only part of the network, were 
analyzed to detect the presence of network subsystems. To this end, the hub nodes were excluded from the correlation matrix, and the remaining correlations were processed by hierarchical cluster analysis using the average linkage algorithm (Everitt et al., 2001). In addition, the network structure emerging from resting-state connectivity data was qualitatively compared to anatomical connectivity data in monkeys (efferent/afferent connections), partially available through the CoCoMac database (Kötter, 2004).

Spatial comparison with the human DMN. We performed a spatial comparison between the monkey and the human DMN, on the basis of the monkey DMN nodes detected by our meta-analysis and of previous results in humans. As no meta-analysis on rewarded fixation in humans is available for a direct comparison with monkey data, we referred for the human DMN to the meta-analysis conducted on fMRI datasets published by Laird et al. (2009). For the definition of the areas in the network, we applied the same procedure as for the monkey DMN. In this case, we used the Colin image registered to the MNI space (as available in MRIcron) for anatomical reference. The cytoarchitectonic classification of human areas was conducted using the SPM Anatomy Toolbox (Eickhoff et al., 2005, 2010; Toga et al., 2006); for the areas left unclassified, we referred to comparative cytoarchitectonic studies (Vogt et al., 1987, 2006; Petrides and Pandya, 1999; Ongür et al., 2003). These procedures have multiple sources of uncertainty, so the correspondences should be taken as best estimates.

\section{Results}

We delineated brain areas potentially belonging to the monkey DMN by performing a meta-analysis of monkey fMRI data (Table 1). Specifically, we calculated a consensus map to define areas with consistent task-induced deactivation across different experiments (single-experiment threshold at $p<0.01, N \geq 8$ ). This analysis revealed a widespread network including the anterior and the posterior cingulate, and the posterior parietal cortices, confirming the existence of a monkey DMN. By overlaying the peaks in the consensus map onto the monkey atlas of Paxinos et al. (2008), we tentatively defined areas 9/46d, 24/32, 8b, caudal temporal parietal occipital (TPOC), 23, 31, v23, PGm, S2i, and PEa to belong to the monkey DMN (Fig. 1, Table 2).

After the definition of the monkey DMN, we examined whether this can be fractionated in functional-anatomical subdivisions, as suggested by human DMN studies (Andrews-Hanna et al., 2010; Sestieri et al., 2011). Based on a methodology already applied on human data (Andrews-Hanna et al., 2010), we measured resting-state connectivity between the monkey DMN nodes to identify in an independent manner the presence of hubs and subsystems. To this end, we used fMRI data collected in four monkeys during fixation (see Table 1). Seed-based functional connectivity analysis showed areas $9 / 46 \mathrm{~d}$ and 31 to be connected to the largest part of the remaining DMN areas (Fig. $2 A, B$ ). The connectivity measures from other DMN nodes, as for example from areas $8 \mathrm{~b}$ (Fig. 3A) and v23 (Fig. 3B), showed only parts of the DMN, suggesting the presence of subsystems.

To test specifically for the existence of subsystems in the DMN, we conducted a connectivity-based network analysis, which allowed us to quantitatively delineate relations among regions. The betweenness-centrality measure (Freeman, 1977) confirmed regions centered at or near areas $9 / 46 \mathrm{~d}$ and 31 to be network hubs and to constitute the DMN core (Fig. 4A). A subset of areas, such as area S2i and PEa, were not found to be significantly correlated with any of the other nodes $(p<0.001)$. By applying cluster analysis on the connected and nonhub nodes, we defined two subsystems. These included areas 24/32, 8b, and TPOC and areas 23, v23, and PGm, respectively (Fig. 4 B). Taskrelated activity profiles revealed the functional selectivity of the

\section{Table 2. List of monkey DMN areas}

\begin{tabular}{|c|c|}
\hline Anatomical label & Coordinates $(x, y, z)$ \\
\hline Areas 24 and 32 in the left anterior cingulate cortex (24/32) & $(5,16,10)$ \\
\hline Areas 24 and 32 in the right anterior cingulate cortex (24/32) & $(-6,15,9)$ \\
\hline Areas 9 and 46d in the left dorsolateral prefrontal cortex (9/46d) & $(-8,15,14)$ \\
\hline Areas 9 and $46 \mathrm{~d}$ in the right dorsolateral prefrontal cortex (9/46d) & $(10,14,13)$ \\
\hline Left area $8 \mathrm{~b}$ in the arcuate sulcus (8b) & $(-10,8,15)$ \\
\hline Right area $8 \mathrm{~b}$ in the arcuate sulcus (8b) & $(10,11,15)$ \\
\hline Left secondary somatosensory area, internal part (S2i) & $(-19,-10,9)$ \\
\hline Right secondary somatosensory area, internal part (S2i) & $(19,-10,10)$ \\
\hline Left area 23 in the posterior cingulate cortex (23) & $(-5,-17,10)$ \\
\hline Right area 23 in the posterior cingulate cortex (23) & $(4,-17,10)$ \\
\hline Left parietal area PEa (PEa) & $(-7,-26,20)$ \\
\hline Right parietal area PEa (PEa) & $(7,-26,21)$ \\
\hline Left area v23 in the posterior cingulate cortex (v23) & $(-7,-28,6)$ \\
\hline Right area v23 in the posterior cingulate cortex (v23) & $(6,-28,8)$ \\
\hline Left temporal parietooccipital area, caudal part (TPOC) & $(-14,-29,16)$ \\
\hline Right temporal parietooccipital area, caudal part (TPOC) & $(14,-29,16)$ \\
\hline Left area 31 in the posterior cingulate cortex (31) & $(-5,-29,10)$ \\
\hline Right area 31 in the posterior cingulate cortex (31) & $(5,-30,11)$ \\
\hline Left parietal area $\mathrm{PG}$, medial part (PGm) & $(-3,-32,14)$ \\
\hline Right parietal area $\mathrm{PG}$, medial part (PGm) & $(4,-32,15)$ \\
\hline
\end{tabular}

For each brain deactivation peak, the anatomical region according to the atlas by Paxinos et al. (2008) and the stereotaxic coordinates in $\mathrm{F} 99$ space are provided.

network core and its subsystems (see the significant differences in percentage signal change across tasks, Fig. 4C).

\section{resting-state connectivity}

\section{A}
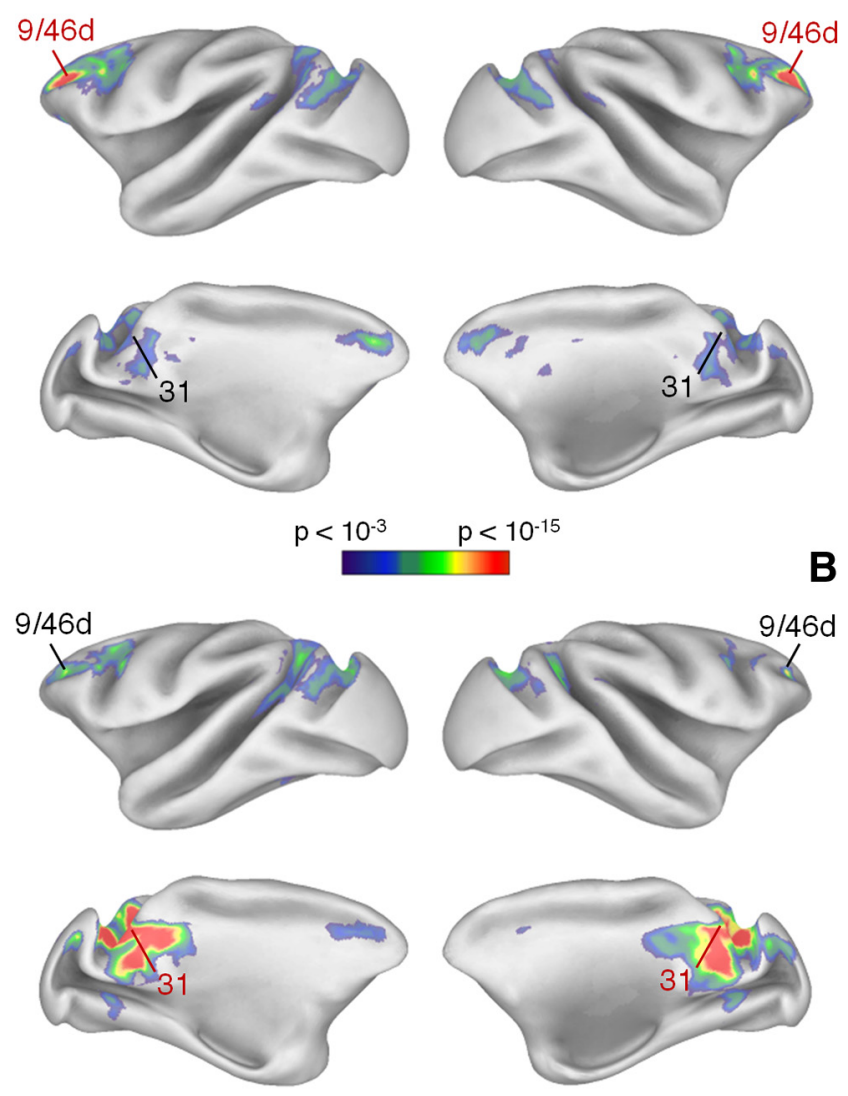

$p<10^{-3} \quad p<10^{-15}$

Figure 2. Resting-state functional connectivity maps from monkey areas $9 / 46 \mathrm{~d}$ and $31 . A, B$, Brain regions showing correlated activity with areas $9 / 46 \mathrm{~d}(\boldsymbol{A})$ and $31(\boldsymbol{B})$ are illustrated on an inflated cortex. The seed area is indicated with the red label. Correlation values are shown using a color scale indicating the statistical significance. 

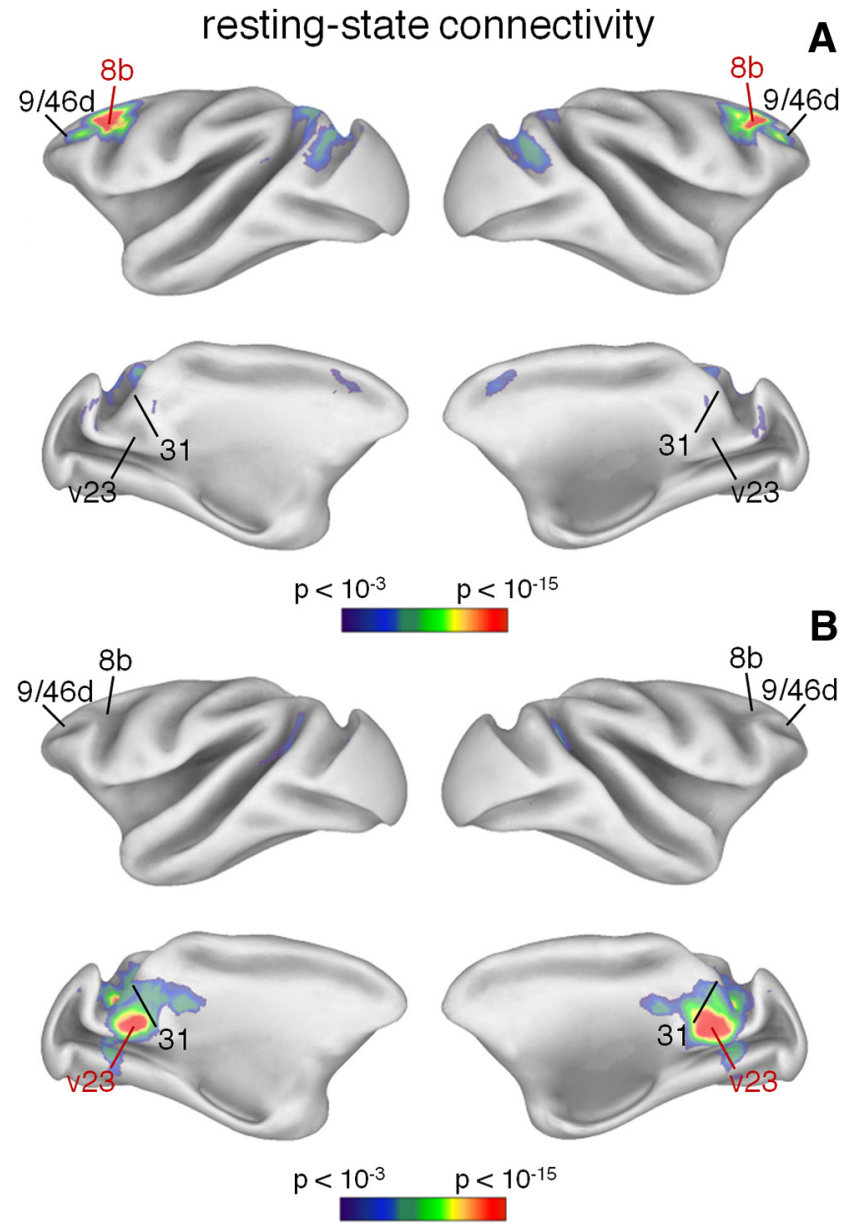

Figure 3. Resting-state functional connectivity maps from monkey areas $8 \mathrm{~b}$ and v23. $\boldsymbol{A}, \boldsymbol{B}$, Brain regions showing correlated activity with areas $8 \mathrm{~b}(\boldsymbol{A})$ and v23b $(\boldsymbol{B})$ are illustrated on an inflated cortex. The seed area is indicated in red. Correlation values are shown in color scale based on the statistical significance.

\section{Discussion}

A previous PET study in monkeys showed regional blood flow increases in the medial prefrontal and posterior cingulate cortex during rest as compared to an active working memory task, providing support for the existence of the monkey DMN (Kojima et al., 2009). Moreover, task-induced suppression of neuronal activity has been shown in the monkey posterior cingulate cortex during attention and working memory tasks (Hayden et al., 2009). By means of our meta-analysis on monkey fMRI data, we defined a set of brain regions that are consistently deactivated across many experimental conditions (Shulman et al., 1997; Gusnard and Raichle, 2001; Mazoyer et al., 2001; Buckner et al., 2008; Laird et al., 2009). This set of areas putatively constitutes a monkey correspondence of the human DMN. Our results are valuable for several reasons: (1) they allow an accurate description of the DMN functional architecture, relying on the large number of anatomical and neurophysiologic studies conducted in monkeys; (2) they show that the DMN is relatively well preserved during $\sim 25$ million years of separate evolution between monkeys and humans; (3) they suggest novel putative functional correspondences between monkey and human brain areas, based on the DMN foci in the two species; and (4) they show that many, though not all, subunits of the DMN are monosynaptically connected with each other.
Network analysis
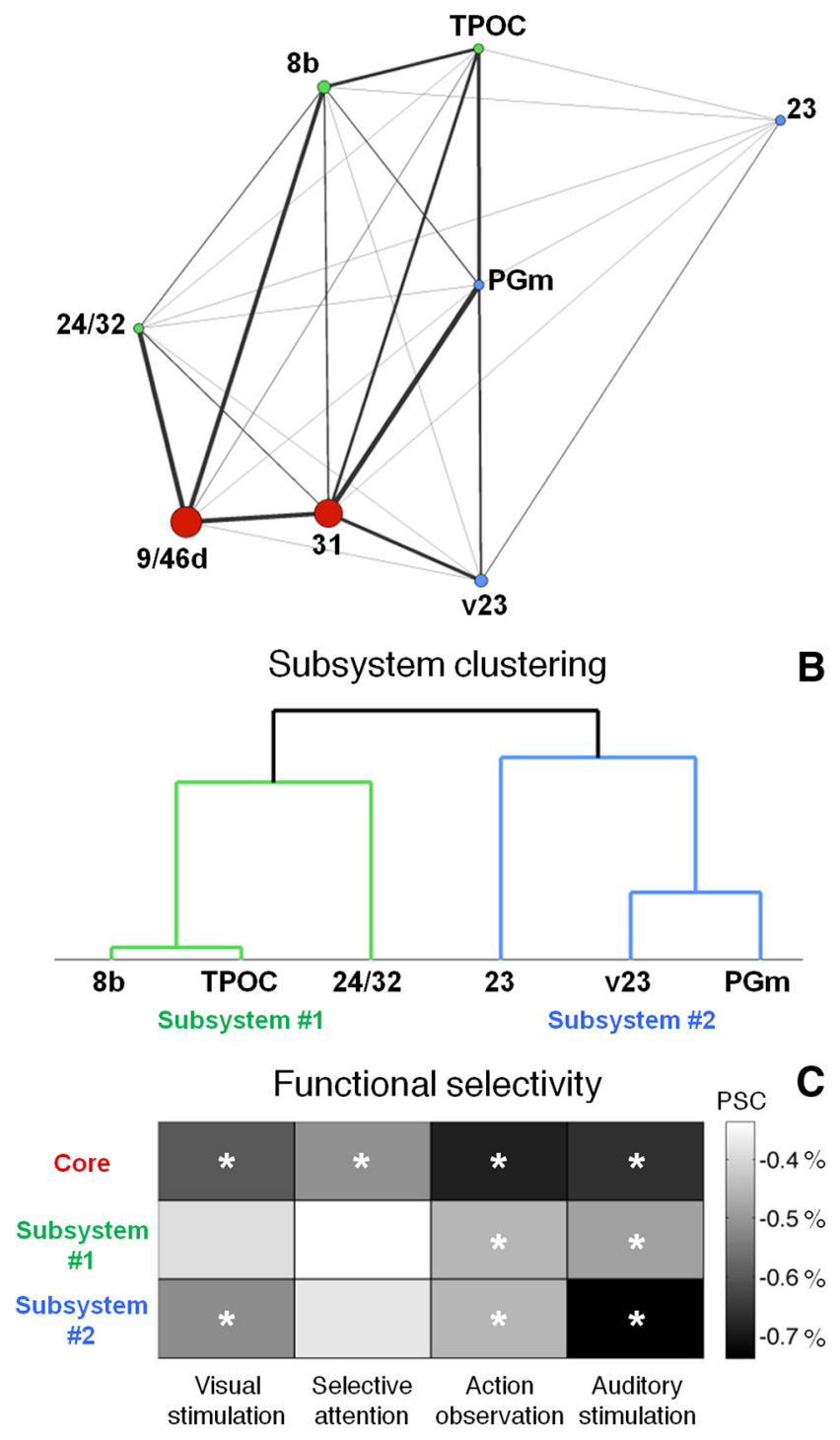

Figure 4. Functional subdivision of the monkey default-mode network. $A$, Network analysis on resting-state connectivity is performed using the Kamada-Kawai algorithm. Only significant correlations at $p<0.001$ are plotted in the graph. The thickness of the lines reflects the strength of the correlation between regions. The size of the circles represents a measure of betweenness-centrality. The two regions with the betweenness-centrality above average, areas $9 / 46$ d and 31 , are considered DMN hubs. B, Hierarchical cluster analysis on the remaining connected regions reveals that the monkey DMN is additionally comprised of two distinct subsystems including areas $24 / 32,8 \mathrm{~b}$, and TPOC and areas $23, \mathrm{~V} 23$, and PGm, respectively. $C$, Selectivity in the deactivation (in percentage signal change, PSC) across multiple experiments is revealed for the network core and its subsystems. The groups of experiments for which the deactivation is significant at $p<0.001$ are marked with asterisks in the plot.

\section{Monkey DMN components}

In this study, the functional organization of the brain areas involved in the DMN was characterized in the monkey using multiple tasks. It is worth mentioning that deactivations from a single experiment may not be sufficient to consistently delineate the DMN, for which instead a meta-analysis across multiple experiments is more reliable (Shulman et al., 1997; Mazoyer et al., 2001; Buckner et al., 2008; Laird et al., 2009). The anatomical definition of the areas in the monkey DMN, classified using a recently published parcellation of the monkey cortex (Paxinos et al., 2008), may permit comparison of our results with the large body of 
Table 3. Anatomical connectivity between monkey DMN areas (Kötter, 2004; Kobayashi and Amaral, 2003; Petrides and Pandya, 2006; Morecraft et al., 2004; Seltzer and Pandya, 2009)

\begin{tabular}{|c|c|c|c|c|c|c|c|c|c|c|}
\hline \multirow[b]{2}{*}{ From } & \multicolumn{10}{|l|}{ To } \\
\hline & $9 / 46 d$ & $24 / 32$ & $8 b$ & TPOC & 31 & 23 & v23 & $\mathrm{PGm}$ & PEa & $\mathrm{S} 2 \mathrm{i}$ \\
\hline $9 / 46 b$ & & $E / A$ & $E / A$ & $\mathrm{E} /-$ & $E / A$ & $\mathrm{E} / \mathrm{A}$ & $E / A$ & $\mathrm{E} /-$ & $-1-$ & $-1-$ \\
\hline $24 / 32$ & & & $\mathrm{E} /-$ & $-1-$ & $\mathrm{E} / \mathrm{A}$ & $\mathrm{E} / \mathrm{A}$ & -1 & $-1-$ & $-1-$ & $-1-$ \\
\hline $8 b$ & & & & $E / A$ & $E / A$ & $E / A$ & $-1-$ & $\mathrm{E} /-$ & $-1-$ & $-1-$ \\
\hline TPOC & & & & & $E / A$ & $E / A$ & $-/ A$ & $-/ A$ & $-1-$ & $-1-$ \\
\hline 31 & & & & & & $E / A$ & $E / A$ & $E / A$ & $-1-$ & $-1-$ \\
\hline 23 & & & & & & & $-/ A$ & $-/ A$ & $E /-$ & $-1-$ \\
\hline V23 & & & & & & & & $E / A$ & $-1-$ & $-1-$ \\
\hline $\mathrm{PGm}$ & & & & & & & & & $-1-$ & $-1-$ \\
\hline PEa & & & & & & & & & & $-1-$ \\
\hline
\end{tabular}

The presence of reported afferent/efferent connections from one area to the other is indicated in the scheme (E, efferent connections; $A$, afferent connections; - , no reported connection). The pairs of areas for which available information from anatomical and resting-state connectivity correspond are presented in bold font. Where there is no correspondence between the two measures, lightface is used. The comparison revealed a correspondence between resting-state and anatomical connectivity for 39 of the 45 analyzed connections. In some cases, such as between areas $24 / 32$ and v23, no anatomical connections were described despite the observed resting-state connectivity. The core areas $9 / 46 \mathrm{~d}$ and 31 were strongly connected with areas 24/32, 8b, TPOC, 23, v23, and PGm, according to both resting-state and anatomical connectivity. Areas in each DMN subsystem (24/32-8b-TPOC and 23-v23-PGm) were more strongly connected with each other and with the core areas, rather than with the areas of the other subsystem. Both connectivity measures showed areas PEa and S2i to be weakly connected with the other DMN nodes.

available anatomical, connectional, and electrophysiological data in the monkey. However, caution is needed with regard to the heterogeneity in areal definitions across studies and inherent uncertainties of the data.

Our results of resting-state connectivity from the monkey DMN areas largely reflect known anatomical connections in the monkey brain, although the match is not perfect (Table 3 ). The monkey DMN, like its human equivalent, consists of a set of interconnected subsystems that converge on hubs including the posterior cingulate cortex (PCC) and regions within prefrontal cortex (PFC) (Buckner et al., 2008; Andrews-Hanna et al., 2010; Sestieri et al., 2011). These hubs correspond with areas $9 / 46 \mathrm{~d}$ and 31 , which are estimated to be anatomically connected to many of the other monkey DMN areas (Barbas and Pandya, 1989; Kobayashi and Amaral, 2003; Morecraft et al., 2004; Parvizi et al., 2006; Petrides and Pandya, 2006, 2007; Price, 2007; Seltzer and Pandya, 2009). Anatomical connectivity data fit well with functional connectivity based on resting-state data of two functionally distinct DMN subsystems, including respectively areas $24 / 32,8$ b, and TPOC (first subsystem) and areas 23, v23, and PGm (second subsystem). Furthermore, not only resting-state based functional connectivity, but also anatomical connectivity showed that areas $\mathrm{PEa}$ and S2i are only weakly connected with the other DMN nodes. In a limited number of cases, we found resting-state connectivity in the absence of documented monosynaptic anatomical connectivity. This may imply either that functional connectivity exists between regions not monosynaptically connected or that anatomical connections exist but have not been described yet (Bullmore and Sporns, 2009), or alternatively may speak to the imperfections in our estimates.

The multisynaptic structure of the monkey DMN and the functional selectivity of its subsystems during goal-directed behavior suggest the network draws on multiple, specialized processing systems. This may explain why there is only partial overlap with the functional connectivity analyses. Functional connectivity analyses are based on correlated fluctuations of fMRI signals (Fox and Raichle, 2007), and therefore they cannot determine the presence of task-related activations or deactivations. Despite the similarity in the medial anterior and posterior areas, our monkey DMN map overlaps only in part with the functional connectivity map originating in the posterior cingulate cortex, as reported by Vincent et al. (2007). It was shown in later analyses of the same data that the choice of different seeds within the posteromedial cortex results in the definition of dis- tinct correlated networks (Margulies et al., 2009). In this regard, our monkey DMN map fits best with the limbic-heteromodal network obtained by Margulies et al. (2009) by seeding specifically the region at or near areas $23 / 31$. It will be interesting to explore further the relationship between network analysis of the monkey systems and their increased activity during passive epochs.

\section{Comparison with the human DMN}

The definition of the human DMN comes from multiple metaanalyses demonstrating consistent task-related deactivations across a network including the PFC, the PCC, and the inferior parietal lobule (Shulman et al., 1997; Mazoyer et al., 2001; Raichle et al., 2001; Buckner et al., 2008; Laird et al., 2009). Both anatomical (Parvizi et al., 2006) and functional (Vincent et al., 2007; Margulies et al., 2009) connectivity investigations focusing on the posterior cingulate cortex suggested correspondent systems the monkey.

To evaluate the potential similarity of the monkey DMN with its human equivalent, we performed a comparative analysis of the deactivation foci between species. To this end, we referred for the human DMN to the results of a previous meta-analysis conducted by activation likelihood estimation on 1711 neuroimaging publications and 7920 experimental contrasts (Laird et al., 2009). We did not carry out quantitative interspecies comparisons, since the tasks performed by monkeys and humans in the two meta-analyses were not the same. The qualitative comparison between spatial maps showed similarities in the broad pattern of deactivated areas (Fig. 5). Putative monkey-human correspondences were primarily found in the vicinity of the medial and dorsolateral prefrontal cortex, the insula/operculum, the lateral parietal cortex, and the posterior cingulate cortex. In both species, the posterior cingulate included multiple foci. It is possible that not only anatomical correspondence, but also functional similarities exist between areas 23/31 of monkeys and humans (Vogt et al., 1987, 2006); however, additional evidence is needed to substantiate such a claim.

We also detected possible interspecies functional similarity in prefrontal cortex for area $8 \mathrm{~b}$, as already predicted by comparative cytoarchitectonic studies (Petrides and Pandya, 1999). It is less clear whether human area OP4, for which a homology with monkey area PV has been proposed (Eickhoff et al., 2010), may instead correspond to monkey area S2i. The monkey DMN areas in PFC correspond only in part to human DMN areas according to 
classical principles of homology and cortical proximity (Orban et al., 2004). For example, monkey area 32 in medial PFC may correspond to two parts in the human brain, including regions $9 / 10 / 32$ ac and 10/24/32 pl, respectively. This result suggests that the prefrontal DMN areas underwent a functional reorganization, in line with the disproportional anatomical expansion of these regions throughout primate evolution (Ongür et al., 2003; Buckner et al., 2008). Interestingly, we also observed deactivations within the posterior-medial intraparietal sulcus in monkeys, but not in humans. Future investigations will be necessary to clarify whether this difference results from the different tasks performed by monkeys and humans or is more directly related to evolutionary changes in the functional architecture of the primate brain.

\section{Putative species-general DMN functions}

Several theories have proposed that the DMN is involved in broad external attention or monitoring. An alternative hypothesis is that the DMN participates in internal modes of cognition evoked spontaneously during passive moments (see Buckner et al., 2008 for a recent review). It is currently unclear which cognitive abilities are present in the monkey and which instead would be exclusively human. The present results suggest that, like humans, monkeys default to consistent sets of processes when left in undirected states. It is tantalizing to infer that monkeys engage in forms of spontaneous cognition detached from the external environment during idle moments. The observation that the anatomy of the DMN in monkeys includes heteromodal association areas and not sensory regions supports this speculative possibility.

In conclusion, our study robustly confirms the existence of a default mode of brain function in monkeys. The topological similarities of the monkey DMN with the human DMN imply a role of this system that generalizes across species, possibly related to internally directed cognition. The DMN may have acquired expanded functions throughout evolution, along with the development of linguistic, self-referential, and social abilities that characterize and distinguish human from nonhuman primates (Schilbach et al., 2008; Passingham, 2009). Further investigations of the monkey DMN areas, for example with intracranial recordings or with fMRI combined with excitatory/inhibitory interventions (e.g., Ekstrom et

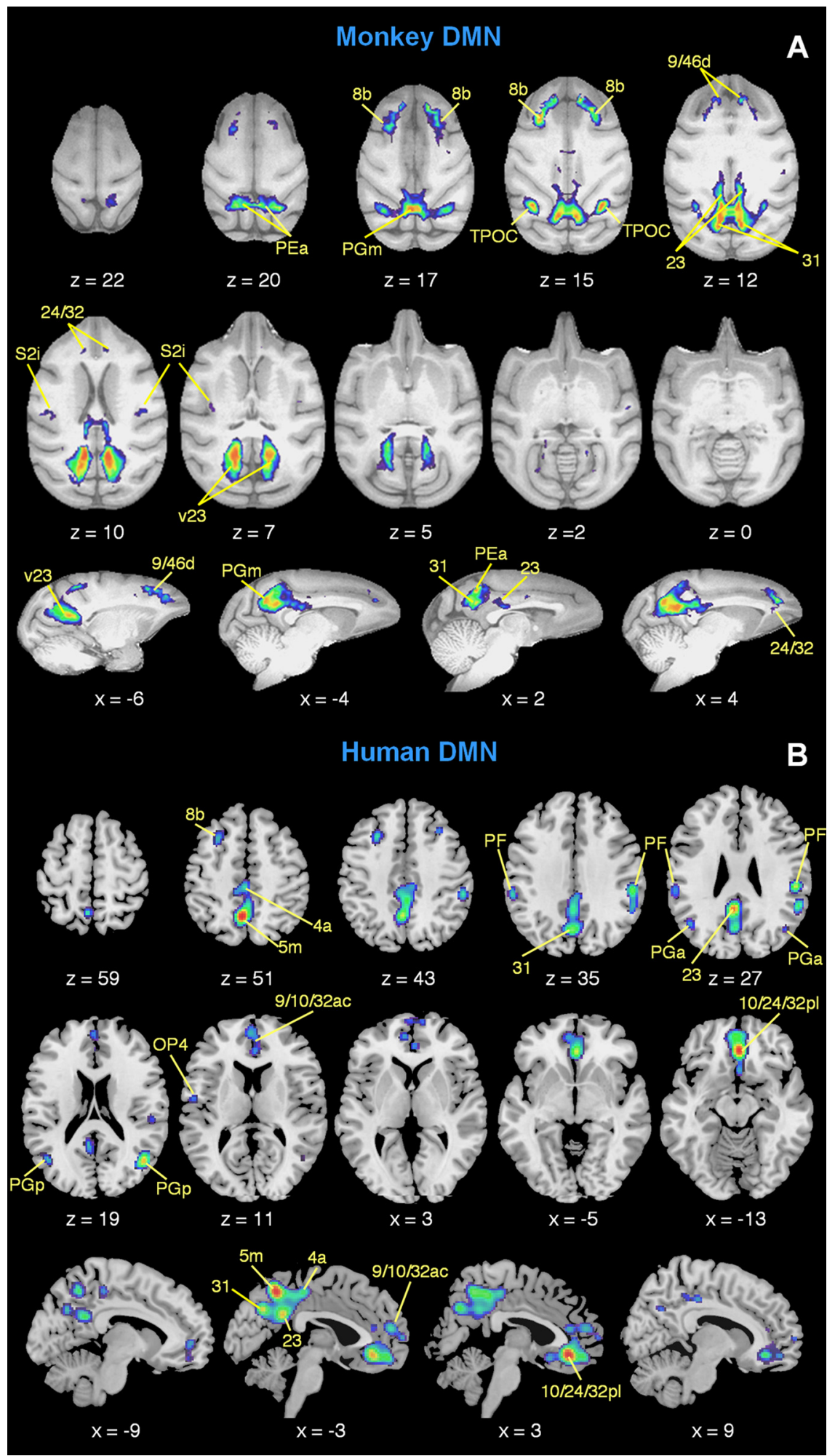

Figure 5. Comparison of monkey $(\boldsymbol{A})$ and human $(\boldsymbol{B})$ default-mode network foci. The monkey DMN obtained from our meta-analysis (as shown in Fig. 1) is spatially compared with a representative human DMN (adapted from Laird et al., 2009). The latter was obtained by a coordinate-based meta-analysis of deactivation peaks across a large number of published human fMRI studies, using the activation likelihood estimation (ALE) method (see Laird et al., 2009 for more details). The ALE map was thresholded at $p<0.005$, corrected with false discovery rate. The monkey and human DMNs are represented on an anatomical template ( $F 99$ and MNI, respectively) in selected axial and sagittal sections. The illustrated foci are classified on the basis of cytoarchitectonic mapping studies. The monkey and human DMNs are spatially comparable, but not necessarily the same in amplitude, as they have been obtained using different datasets and different statistical approaches. 
al., 2008), are needed to evaluate the electrophysiological and behavioral correlates of the default-mode activity and to better understand the functional role of the DMN.

\section{References}

Andrews-Hanna JR, Reidler JS, Sepulcre J, Poulin R, Buckner RL (2010) Functional-anatomic fractionation of the brain's default network. Neuron 65:550-562.

Barbas H, Pandya DN (1989) Architecture and intrinsic connections of the prefrontal cortex in the rhesus monkey. J Comp Neurol 286:353-375.

Binder JR, Desai RH, Graves WW, Conant LL (2009) Where is the semantic system? A critical review and meta-analysis of 120 functional neuroimaging studies. Cereb Cortex 19:2767-2796.

Buckner RL, Andrews-Hanna JR, Schacter DL (2008) The brain's default network: anatomy, function, and relevance to disease. Ann N Y Acad Sci 1124:1-38.

Buckner RL, Sepulcre J, Talukdar T, Krienen FM, Liu H, Hedden T, AndrewsHanna JR, Sperling RA, Johnson KA (2009) Cortical hubs revealed by intrinsic functional connectivity: mapping, assessment of stability, and relation to Alzheimer's disease. J Neurosci 29:1860-1873.

Bullmore E, Sporns O (2009) Complex brain networks: graph theoretical analysis of structural and functional systems. Nat Rev Neurosci 10:186-198.

Caggiano V, Fogassi L, Rizzolatti G, Thier P, Casile A (2009) Mirror neurons differentially encode the peripersonal and extrapersonal space of monkeys. Science 324:403-406.

Caminiti R, Chafee MV, Battaglia-Mayer A, Averbeck BB, Crowe DA, Georgopoulos AP (2010) Understanding the parietal lobe syndrome from a neurophysiological and evolutionary perspective. Eur J Neurosci 31:2320-2340.

Durand JB, Nelissen K, Joly O, Wardak C, Todd JT, Norman JF, Janssen P, Vanduffel W, Orban GA (2007) Anterior regions of monkey parietal cortex process visual 3D shape. Neuron 55:493-505.

Eickhoff SB, Stephan KE, Mohlberg H, Grefkes C, Fink GR, Amunts K, Zilles K (2005) A new SPM toolbox for combining probabilistic cytoarchitectonic maps and functional imaging data. Neuroimage 25:1325-1335.

Eickhoff SB, Jbabdi S, Caspers S, Laird AR, Fox PT, Zilles K, Behrens TE (2010) Anatomical and functional connectivity of cytoarchitectonic areas within the human parietal operculum. J Neurosci 30:6409-6421.

Ekstrom LB, Roelfsema PR, Arsenault JT, Bonmassar G, Vanduffel W (2008) Bottom-up dependent gating of frontal signals in early visual cortex. Science 321:414-417.

Everitt BS, Landau S, Leese M (2001) Cluster analysis. New York: Oxford UP.

Fox MD, Raichle ME (2007) Spontaneous fluctuations in brain activity observed with functional magnetic resonance imaging. Nat Rev Neurosci $8: 700-711$.

Freeman LC (1977) A set of measures of centrality based on betweenness. Sociometry 40:35-41.

Frey S, Pandya DN, Chakravarty MM, Bailey L, Petrides M, Collins DL (2011) An MRI based average macaque monkey stereotaxic atlas and space (MNI monkey space). Neuroimage 55:1435-1442.

Genest C (1992) Vincentization revisited. Ann Stat 20:1137-1142.

Greicius MD, Krasnow B, Reiss AL, Menon V (2003) Functional connectivity in the resting brain: a network analysis of the default mode hypothesis. Proc Natl Acad Sci U S A 100:253-258.

Gusnard DA, Raichle ME (2001) Searching for a baseline: functional imaging and the resting human brain. Nat Rev Neurosci 2:685-694.

Hayden BY, Smith DV, Platt ML (2009) Electrophysiological correlates of default-mode processing in macaque posterior cingulate cortex. Proc Natl Acad Sci U S A 106:5948-5953.

Joly O, Ramus F, Pressnitzer D, Vanduffel W, Orban GA (2011) Interhemispheric differences in auditory processing revealed by fMRI in awake rhesus monkeys. Cereb Cortex. Advance online publication. doi:10.1093/cercor/bhr150.

Kamada T, Kawai S (1989) An algorithm for drawing general undirected graphs. Inf Process Lett 31:7-15.

Kobayashi Y, Amaral DG (2003) Macaque monkey retrosplenial cortex: II. Cortical afferents. J Comp Neurol 466:48-79.

Kojima T, Onoe H, Hikosaka K, Tsutsui K, Tsukada H, Watanabe M (2009) Default mode of brain activity demonstrated by positron emission tomography imaging in awake monkeys: higher rest-related than working memory-related activity in medial cortical areas. J Neurosci 29:1446314471.

Kötter R (2004) Online retrieval, processing, and visualization of primate connectivity data from the CoCoMac database. Neuroinformatics 2: 127-144.

Laird AR, Eickhoff SB, Li K, Robin DA, Glahn DC, Fox PT (2009) Investigating the functional heterogeneity of the default mode network using coordinate-based meta-analytic modeling. J Neurosci 29:14496-14505.

Margulies DS, Vincent JL, Kelly C, Lohmann G, Uddin LQ, Biswal BB, Villringer A, Castellanos FX, Milham MP, Petrides M (2009) Precuneus shares intrinsic functional architecture in humans and monkeys. Proc Natl Acad Sci U S A 106:20069-20074.

Mazoyer B, Zago L, Mellet E, Bricogne S, Etard O, Houdé O, Crivello F, Joliot M, Petit L, Tzourio-Mazoyer N (2001) Cortical networks for working memory and executive functions sustain the conscious resting state in man. Brain Res Bull 54:287-298.

Moeller S, Freiwald WA, Tsao DY (2008) Patches with links: a unified system for processing faces in the macaque temporal lobe. Science 320:1355-1359.

Morecraft RJ, Cipolloni PB, Stilwell-Morecraft KS, Gedney MT, Pandya DN (2004) Cytoarchitecture and cortical connections of the posterior cingulate and adjacent somatosensory fields in the rhesus monkey. J Comp Neurol 469:37-69.

Nakahara K, Hayashi T, Konishi S, Miyashita Y (2002) Functional MRI of macaque monkeys performing a cognitive set-shifting task. Science 295:1532-1536.

Nelissen K, Luppino G, Vanduffel W, Rizzolatti G, Orban GA (2005) Observing others: multiple action representation in the frontal lobe. Science 310:332-336

Nelissen K, Vanduffel W, Orban GA (2006) Charting the lower superior temporal region, a new motion-sensitive region in monkey superior temporal sulcus. J Neurosci 26:5929-5947.

Nelissen K, Joly O, Durand JB, Todd JT, Vanduffel W, Orban GA (2009) The extraction of depth structure from shading and texture in the macaque brain. PLoS One 4:e8306.

Ongür D, Ferry AT, Price JL (2003) Architectonic subdivision of the human orbital and medial prefrontal cortex. J Comp Neurol 460:425-449.

Orban GA, Van Essen D, Vanduffel W (2004) Comparative mapping of higher visual areas in monkeys and humans. Trends Cogn Sci 8:315-324.

Parvizi J, Van Hoesen GW, Buckwalter J, Damasio A (2006) Neural connections of the posteromedial cortex in the macaque. Proc Natl Acad Sci U S A 103:1563-1568.

Passingham R (2009) How good is the macaque monkey model of the human brain? Curr Opin Neurobiol 19:6-11.

Paxinos G, Huang XF, Petrides M, Toga AW (2008) The rhesus monkey brain in stereotactic coordinates. London, UK: Elsevier Academic.

Peeters R, Simone L, Nelissen K, Fabbri-Destro M, Vanduffel W, Rizzolatti G, Orban GA (2009) The representation of tool use in humans and monkeys: common and uniquely human features. J Neurosci 29:11523-11539.

Petrides M, Pandya DN (1999) Dorsolateral prefrontal cortex: comparative cytoarchitectonic analysis in the human and the macaque brain and corticocortical connection patterns. Eur J Neurosci 11:1011-1136.

Petrides M, Pandya DN (2006) Efferent association pathways originating in the caudal prefrontal cortex in the macaque monkey. J Comp Neurol 498:227-251

Petrides M, Pandya DN (2007) Efferent association pathways from the rostral prefrontal cortex in the macaque monkey. J Neurosci 27:11573-11586

Price JL (2007) Definition of the orbital cortex in relation to specific connections with limbic and visceral structures and other cortical regions. Ann N Y Acad Sci 1121:54-71.

Raichle ME, MacLeod AM, Snyder AZ, Powers WJ, Gusnard DA, Shulman GL (2001) A default mode of brain function. Proc Natl Acad Sci U S A 98:676-682.

Sawamura H, Georgieva S, Vogels R, Vanduffel W, Orban GA (2005) Using functional magnetic resonance imaging to assess adaptation and size invariance of shape processing by humans and monkeys. J Neurosci 25:4294-4306

Schilbach L, Eickhoff SB, Rotarska-Jagiela A, Fink GR, Vogeley K (2008) Minds at rest? Social cognition as the default mode of cognizing and its putative relationship to the "default system" of the brain. Conscious Cogn 17:457-467. 
Seltzer B, Pandya DN (2009) Posterior cingulate and retrosplenial cortex connections of the caudal superior temporal region in the rhesus monkey. Exp Brain Res 195:325-334.

Sestieri C, Corbetta M, Romani GL, Shulman GL (2011) Episodic memory retrieval, parietal cortex, and the default mode network: functional and topographic analyses. J Neurosci 31:4407-4420.

Shulman GL, Fiez JA, Corbetta M, Buckner RL, Miezin FM, Raichle ME, Petersen SE (1997) Common blood flow changes across visual tasks: II. Decreases in cerebral cortex. J Cogn Neurosci 9:648-663.

Shumway RH, Stoffer DS (2006) Time series analysis and its applications. New York: Springer.

Toga AW, Thompson PM, Mori S, Amunts K, Zilles K (2006) Towards multimodal atlases of the human brain. Nat Rev Neurosci 7:952-966.

Tsao DY, Freiwald WA, Tootell RB, Livingstone MS (2006) A cortical region consisting entirely of face-selective cells. Science 311:670-674.

Tsao DY, Moeller S, Freiwald WA (2008) Comparing face patch systems in macaques and humans. Proc Natl Acad Sci U S A 105:19514-19519.

Vaishnavi SN, Vlassenko AG, Rundle MM, Snyder AZ, Mintun MA, Raichle ME (2010) Regional aerobic glycolysis in the human brain. Proc Natl Acad Sci U S A 107:17757-17762.

Vanduffel W, Fize D, Mandeville JB, Nelissen K, Van Hecke P, Rosen BR,
Tootell RB, Orban GA (2001) Visual motion processing investigated using contrast agent-enhanced fMRI in awake behaving monkeys. Neuron 32:565-577.

Vanduffel W, Fize D, Peuskens H, Denys K, Sunaert S, Todd JT, Orban GA (2002) Extracting 3D from motion: differences in human and monkey intraparietal cortex. Science 298:413-415.

Vincent JL, Patel GH, Fox MD, Snyder AZ, Baker JT, Van Essen DC, Zempel JM, Snyder LH, Corbetta M, Raichle ME (2007) Intrinsic functional architecture in the anaesthetized monkey brain. Nature 447:83-86.

Vincent JL, Kahn I, Van Essen DC, Buckner RL (2010) Functional connectivity of the macaque posterior parahippocampal cortex. J Neurophysiol 103:793-800.

Vogt BA, Pandya DN, Rosene DL (1987) Cingulate cortex of the rhesus monkey: I. Cytoarchitecture and thalamic afferents. J Comp Neurol 262:256-270.

Vogt BA, Vogt L, Laureys S (2006) Cytology and functionally correlated circuits of human posterior cingulate areas. Neuroimage 29:452-466.

Wardak C, Vanduffel W, Orban GA (2010) Searching for a salient target involves frontal regions. Cereb Cortex 20:2464-2477.

Zar JH (1998) Biostatistical analysis. Upper Saddle River, NJ: Prentice-Hall. 